\title{
SOME FIXED POINT THEOREM USING COMMON PROPERTY(E.A.) IN INTUITIONISTIC FUZZY METRIC SPACE
}

\author{
JONG SEO PARK
}

\begin{abstract}
In this paper, we define the intuitionistic fuzzy contraction, and obtain some fixed point theorem using common property(E.A.) and weakly compatibility in intuitionistic fuzzy metric space.
\end{abstract}

\section{INTRODUCTION}

George and Veeramani [1] modified the concept of fuzzy metric space introduced by Kramosil and Michalek [4] with a view to obtain a Hausdorff topology on fuzzy metric spaces, and this has recently found very fruitful applications in quantum particle physics. In recent years, many authors have proved fixed point theorems in fuzzy metric spaces, and we observed some common fixed point theorems in fuzzy metric space which improved many known results ([2], [9], [11] etc).

Sessa[10] introduced the notion of weakly commuting mappings which was further enlarged by Jungck[3] by defining compatible mapping in fuzzy metric space. Also, Park[6] studied some properties for compatible map in intuitionistic fuzzy metric space.

In this paper, we define the intuitionistic fuzzy contraction, and obtain some fixed point theorem using common property(E.A.) and weakly compatibility in intuitionistic fuzzy metric space.

\section{Preliminaries}

We recall some definitions, properties and known results in the intuitionistic fuzzy metric space as following :

Received by the editors April 11, 2011. Accepted August 4, 2011. 2000 Mathematics Subject Classification. 46S40, 47H10, 54H25.

Key words and phrases. weakly compatibility, fixed point, property(E.A.). 
Let us recall (see [8]) that a continuous $t$-norm is a operation $*:[0,1] \times[0,1] \rightarrow$ $[0,1]$ which satisfies the following conditions: (a)* is commutative and associative, (b)* is continuous, (c) $a * 1=a$ for all $a \in[0,1]$, (d) $a * b \leq c * d$ whenever $a \leq c$ and $b \leq d(a, b, c, d \in[0,1])$. Also, a continuous $t$-conorm is a operation $\diamond$ : $[0,1] \times[0,1] \rightarrow[0,1]$ which satisfies the following conditions: $(a) \diamond$ is commutative and associative, (b) $\diamond$ is continuous, (c) $a \diamond 0=a$ for all $a \in[0,1]$, (d) $a \diamond b \geq c \diamond d$ whenever $a \leq c$ and $b \leq d(a, b, c, d \in[0,1])$.

Definition $2.1([5])$. The 5 -tuple $(X, M, N, *, \diamond)$ is said to be an intuitionistic fuzzy metric space if $X$ is an arbitrary set, $*$ is a continuous $t$-norm, $\diamond$ is a continuous $t$-conorm and $M, N$ are fuzzy sets on $X^{2} \times(0, \infty)$ satisfying the following conditions; for all $x, y, z \in X$, such that
(a) $M(x, y, t)>0$,
(b) $M(x, y, t)=1$ if and only if $x=y$,
(c) $M(x, y, t)=M(y, x, t)$,
(d) $M(x, y, t) * M(y, z, s) \leq M(x, z, t+s)$,
(e) $M(x, y, \cdot):(0, \infty) \rightarrow(0,1]$ is continuous,
(f) $N(x, y, t)>0$,
(g) $N(x, y, t)=0$ if and only if $x=y$,
(h) $N(x, y, t)=N(y, x, t)$,
(i) $N(x, y, t) \diamond N(y, z, s) \geq N(x, z, t+s)$,
(j) $N(x, y, \cdot):(0, \infty) \rightarrow(0,1]$ is continuous.

Note that $(M, N)$ is called an intuitionistic fuzzy metric on $X$. The functions $M(x, y, t)$ and $N(x, y, t)$ denote the degree of nearness and the degree of non-nearness between $x$ and $y$ with respect to $t$, respectively.

Definition $2.2([7])$. Let $X$ be an intuitionistic fuzzy metric space.

(a) $\left\{x_{n}\right\}$ is said to be convergent to a point $x \in X$ by $\lim _{n \rightarrow \infty} x_{n}=x$ if $\lim _{n \rightarrow \infty} M\left(x_{n}, x, t\right)=1, \lim _{n \rightarrow \infty} N\left(x_{n}, x, t\right)=0$ for all $t>0$.

(b) $\left\{x_{n}\right\}$ is called a Cauchy sequence if

$$
\lim _{n \rightarrow \infty} M\left(x_{n+p}, x_{n}, t\right)=1, \quad \lim _{n \rightarrow \infty} N\left(x_{n+p}, x_{n}, t\right)=0
$$

for all $t>0$ and $p>0$.

(c) $X$ is complete if every Cauchy sequence converges in $X$.

Definition $2.3([6])$. A pair of self mappings $(f, g)$ defined on an intuitionistic 
fuzzy metric space $X$ is said to be compatible (or asymptotically commuting) if for all $t>0$

$$
\lim _{n \rightarrow \infty} M\left(f g x_{n}, g f x_{n}, t\right)=1, \quad \lim _{n \rightarrow \infty} N\left(f g x_{n}, g f x_{n}, t\right)=0
$$

where $\left\{x_{n}\right\} \subset X$ such that $\lim _{n \rightarrow \infty} f x_{n}=\lim _{n \rightarrow \infty} g x_{n}=z$ for some $z \in X$. Also, the pair $(f, g)$ is called non-compatible, if there exists a sequence $\left\{x_{n}\right\} \subset X$ such that $\lim _{n \rightarrow \infty} f x_{n}=\lim _{n \rightarrow \infty} g x_{n}=z$, but either $\lim _{n \rightarrow \infty} M\left(f g x_{n}, g f x_{n}, t\right) \neq$ 1, $\lim _{n \rightarrow \infty} N\left(f g x_{n}, g f x_{n}, t\right) \neq 0$ or the limit does not exists.

Definition 2.4. A pair of self mappings $(f, g)$ defined on an intuitionistic fuzzy metric space $X$ is said to satisfy the property(E.A.) if there exists a sequence $\left\{x_{n}\right\} \subset$ $X$ such that $\lim _{n \rightarrow \infty} f x_{n}=\lim _{n \rightarrow \infty} g x_{n}=z$ for some $z \in X$.

Definition 2.5. Two pairs of self mappings $(A, S)$ and $(B, T)$ defined on an intuitionistic fuzzy metric space $X$ are said to share common property(E.A.) if there exist sequences $\left\{x_{n}\right\},\left\{y_{n}\right\} \subset X$ such that $\lim _{n \rightarrow \infty} A x_{n}=\lim _{n \rightarrow \infty} S x_{n}=\lim _{n \rightarrow \infty} B y_{n}=$ $\lim _{n \rightarrow \infty} T y_{n}=z$ for some $z \in X$.

Definition $2.6([6])$. Two self mappings $f$ and $g$ on an intuitionistic fuzzy metric space $X$ are called weakly compatible if they commute at their point of coincidence. That is, $f x=g x$ implies $f g x=g f x$.

Definition 2.7. Let $X$ be an intuitionistic fuzzy metric space and $(f, g)$ be a pair of maps from $X$ into $X$. The map $f$ is called an intuitionistic fuzzy contraction with respect to $g$ if there exists an upper semicontinuous function $r:[0, \infty) \rightarrow[0, \infty)$ with $r(\tau)<\tau$ for every $\tau>0$ such that

$$
\begin{aligned}
& \frac{1}{M(f x, f y, t)}-1 \leq r\left(\frac{1}{m(f, g, x, y, t)}-1\right), \\
& N(f x, f y, t) \leq r(n(f, g, x, y, t))
\end{aligned}
$$

for every $x, y \in X$ and each $t>0$, where

$$
\begin{aligned}
& m(f, g, x, y, t)=\min \{M(g x, g y, t), M(f x, g x, t), M(f y, g y, t)\}, \\
& n(f, g, x, y, t)=\max \{N(g x, g y, t), N(f x, g x, t), N(f y, g y, t)\} .
\end{aligned}
$$

Definition 2.8. Let $A, B, S$ and $T$ be four self mappings of an intuitionistic fuzzy metric space $X$. Then the mappings $A$ and $B$ are called a generalized intuitionistic fuzzy contraction with respect to $S$ and $T$ if there exists an upper semicontinuous function $r:[0, \infty) \rightarrow[0, \infty)$ with $r(\tau)<\tau$ for every $\tau>0$ such that for each 
$x, y \in X$ and $t>0$,

$$
\begin{aligned}
& \quad \frac{1}{M(A x, B y, t)}-1 \\
& \quad \leq r\left(\frac{1}{\min \{M(S x, T y, t), M(A x, S x, t), M(B y, T y, t)\}}-1\right), \\
& N(A x, B y, t) \\
& \quad \leq r(\max \{N(S x, T y, t), N(A x, S x, t), N(B y, T y, t)\}) .
\end{aligned}
$$

\section{Main Result}

In this part, we prove some common fixed point theorem for four mappings satisfying weak compatible using property(E.A.).

Theorem 3.1. Let $A, B, S$ and $T$ be four self mappings of an intuitionistic fuzzy metric space $X$ such that the mappings $A$ and $B$ are generalized intuitionistic fuzzy contraction with respect to mappings $S$ and $T$. Suppose that the pairs $(A, S)$ and $(B, T)$ share the common property(E.A.) and $S(X), T(X)$ are closed subsets of $X$. Then the pair $(A, S)$ as well as $(B, T)$ have a point of coincidence each. Furthermore, if the pairs $(A, S)$ and $(B, T)$ are weakly compatible, then $A, B, S$ and $T$ have a unique common fixed point.

Proof. By assumption, since $(A, S)$ and $(B, T)$ share the common property(E.A.), there exist sequences $\left\{x_{n}\right\},\left\{y_{n}\right\} \subset X$ such that for some $z \in X, \lim _{n \rightarrow \infty} A x_{n}=$ $\lim _{n \rightarrow \infty} S x_{n}=\lim _{n \rightarrow \infty} B y_{n}=\lim _{n \rightarrow \infty} T y_{n}=z$. Since $S(X)$ is a closed subset of $X, \lim _{n \rightarrow \infty} S x_{n}=z \in S(X)$. Therefore, there exist a point $u \in X$ such that $S u=z$.

Now, we prove $S u=A u$. If not, then by (2.1), we have

$$
\begin{aligned}
& \quad \frac{1}{M\left(A u, B y_{n}, t\right)}-1 \\
& \quad \leq r\left(\frac{1}{\min \left\{M\left(S u, T y_{n}, t\right), M(A u, S u, t), M\left(B y_{n}, T y_{n}, t\right)\right\}}-1\right), \\
& N\left(A u, B y_{n}, t\right) \\
& \quad \leq r\left(\max \left\{N\left(S u, T y_{n}, t\right), N(A u, S u, t), N\left(B y_{n}, T y_{n}, t\right)\right\}\right) .
\end{aligned}
$$

Hence for every $t>0$, as $n \rightarrow \infty$,

$$
\begin{aligned}
& \frac{1}{M(A u, z, t)}-1 \leq r\left(\frac{1}{\min \{M(A u, z, t)\}}-1\right)<\frac{1}{M(A u, z, t)}-1, \\
& N(A u, z, t) \leq r(\max \{N(A u, z, t)\})<N(A u, z) .
\end{aligned}
$$


This is a contradiction, and hence $S u=A u$. Therefore $u$ is a coincidence point of the pair $(A, S)$.

If $T(X)$ is a closed subset of $X$, then $\lim _{n \rightarrow \infty} T y_{n}=z \in T(X)$. That is, there exist a point $v \in X$ such that $T v=z$. Now, we prove $T v=B v$. If not, then we have

$$
\begin{aligned}
& \quad \frac{1}{M(A x, B v, t)}-1 \\
& \quad \leq r\left(\frac{1}{\min \left\{M\left(S x_{n}, T v, t\right), M\left(A x_{n}, S x_{n}, t\right), M(B v, T v, t)\right\}}-1\right), \\
& N\left(A x_{n}, B v, t\right) \\
& \quad \leq r\left(\max \left\{N\left(S x_{n}, T v, t\right), N\left(A x_{n}, S x_{n}, t\right), N(B v, T v, t)\right\}\right) .
\end{aligned}
$$

Hence for every $t>0$ as $n \rightarrow \infty$,

$$
\begin{aligned}
& \frac{1}{M(z, B v, t)}-1 \leq r\left(\frac{1}{\min \{M(z, B v, t)\}}-1\right)<\frac{1}{M(z, B v, t)}-1, \\
& N(z, B v, t) \leq r(\max \{N(z, B v, t)\})<N(z, B v, t) .
\end{aligned}
$$

This is a contradiction, and hence $B v=T v$. Therefore $v$ is a coincidence point of the pair $(B, T)$. Since $(A, S)$ is weakly compatible and $A u=S u$, we have $A z=$ $A S u=S A u=S z$.

Suppose that $A z \neq z$, then by (2.1), we have for all $t>0$,

$$
\begin{aligned}
& \frac{1}{M(A z, B v, t)}-1 \leq r\left(\frac{1}{\min \{M(A z, B v, t)\}}-1\right)<\frac{1}{M(A z, B v, t)}-1, \\
& N(A z, B v, t) \leq r(\max \{N(A z, B v, t)\})<N(A z, B v, t) .
\end{aligned}
$$

Hence $A z=B v=z$ from contradiction. By same method for the pair $(B, T)$, we get $B z=T v=z$. Therefore $z$ is a common fixed point of the pairs $(A, S)$ and $(B, T)$.

Uniqueness of the common fixed point $z$ is an easy consequence of (2.1). This completes the proof.

Example 3.2. Let $X=[2,20]$ and $X$ be an intuitionistic fuzzy metric space defined $M, N$ as $M(x, y, t)=\frac{t}{t+d(x, y)}, N(x, y, t)=\frac{d(x, y)}{t+d(x, y)}$ for all $x, y \in X$ and $t>0$. Define $A, B, S, T: X \rightarrow X$ by

$$
A x=\left\{\begin{array}{ll}
2 & \text { if } x=2, \\
3 & \text { if } x>2,
\end{array} \quad B x= \begin{cases}2 & \text { if } x=2, \\
6 & \text { if } 2<x \leq 5, \\
3 & \text { if } x>5,\end{cases}\right.
$$




$$
S x=\left\{\begin{array}{ll}
2 & \text { if } x=2, \\
6 & \text { if } x>2,
\end{array} \quad T x= \begin{cases}2 & \text { if } x=2, \\
18 & \text { if } 2<x \leq 5, \\
12 & \text { if } x>5,\end{cases}\right.
$$

Then $A, B, S$ and $T$ satisfy all conditions of Theorem 3.1 with $r(\tau)=k \tau$, where $k \in\left(\frac{4}{9}, 1\right)$. Hence 2 is a unique common fixed point of $A, B, S$ and $T$ which also remains a point of discontinuity.

\section{REFERENCES}

1. George, A. \& Veeramani, P.: On some results of analysis for fuzzy metric spaces. Fuzzy Sets and Systems 90 (1997), 365-368.

2. Imdad, M. \& Ali, J.: Some common fixed point theorems in fuzzy metric spaces. Math. Commun. 11 (2006), 153-163.

3. Jungck, G.: Compatible mappings and common fixed points. Int. J. Math. Math. Sci. 9 (1986), 771-779.

4. Kramosil, J. \& Michalek, J.: Fuzzy metric and statistical metric spaces. Kybernetica 11 (1975), 326-334.

5. Park, J.H., Park, J.S. \& Kwun, Y.C.: A common fixed point theorem in the intuitionistic fuzzy metric space. Advances in Natural Comput. Data Mining(Proc. 2nd ICNC and 3rd FSKD) (2006), 293-300.

6. Park, J.S.: On fixed point theorem of weak compatible maps of type $(\gamma)$ in complete intuitionistic fuzzy metric space. Int. J. F. I. S. 11 (2011), no. 1, 38-43.

7. Park, J.S., Park, J.H. \& Kwun, Y.C.: On some results for five mappings using compatibility of type $(\alpha)$ in intuitionistic fuzzy metric space. International J. Fuzzy Logic Intelligent Systems 8 (2008), no. 4, 299-305.

8. Schweizer, B. \& Sklar, A.: Statistical metric spaces. Pacific J. Math. 10 (1960), 314-334.

9. Singh, B. \& Jain, S.: Weak compatibility and fixed point theorems in fuzzy metric space. Ganita 56 (2005), 167-176.

10. Sessa, S.: 1982. On a weak commutatively condition in fixed point considerations. Publ. Inst. Math. 32 (1982), 149-153.

11. Vetro, C. \& Vetro, P.: Common fixed points for discontinuous mappings in fuzzy metric space. Rend. Circ. Mat. Palermo 57 (2008), 295-303.

Department of Mathematics Education, Chinju National University of Education, Jinju 660-756, KoREA

Email address: parkjs@cue.ac.kr 